\title{
Breaker to Control Center Integrated Protection, Control and Operations Model
}

\author{
Sakis Meliopoulos ${ }^{1}$ \\ Sakis.m@gatech.edu
}

Mohsen Pilehvar ${ }^{1}$

pilehvar@ksu.edu

${ }^{1}$ Georgia Institute of Technology

\author{
Siyao Cai ${ }^{1}$ \\ scai37@gatech.edu \\ Gad Monga Ilunga ${ }^{1}$
}

George Cokkinides ${ }^{1}$

cokkinides@comcast.net
Paul Myrda
pmyrda@epri.com
Evangelos Farantatos ${ }^{2}$ efarantatos@epri.com

${ }^{2}$ Electric Power Research Institute

\begin{abstract}
Technological advances in electric energy system data acquisition systems, time synchronization, and cyber assets used in power system substations, distribution systems, and control centers offer new opportunities to dramatically improve the practice of monitoring, protection, control, and operation of the system. We can make the computer based new technologies smarter and more intelligent to fully automate the basic protection and control functions. The challenges posed to the system from the continuous deployment of renewable resources that are typically inverter interface resources require monitoring of the system at much higher rates and development of protection and control systems that can respond in much faster rates than for conventional systems and they are immune to the characteristics of the new system, namely reduced fault currents and suppressed negative and zero sequence components of the fault currents. We propose a new system that provides validated data at fast rates (once per cycle), protective relays that are immune to the effects of inverter interfaced generation, detect anomalies, and enable the continuous operation of relays and other functions even in the presence of hidden failures in instrumentation. This system will enable the operators to meet the challenges posed by the evolving power system and provides robust solutions to the new requirements.
\end{abstract}

\section{Introduction}

Power systems are experiencing a fast transformation from the integration of renewables, new technologies for metering, protection and control, and fast occurring transients that challenge the present protection control and operation systems. We have seen usual fault events which were cleared within typical relay response times (few cycles), but because of the interaction of the fault transients and the control characteristics of inverter interfaced resources, substantial amounts of renewable resources were lost. These events are sounding the alarm that the power system of the future will be vulnerable to massive loss of renewables from typical fault events that have not caused any concern for the legacy power system.

Additional challenges exist. At the protection level, the new characteristics affect the operation of legacy protection systems. Specific issues that have been identified are: high penetration levels of renewables affect the levels of faults currents and the characteristics of fault currents with reduced negative and zero sequence components. This, in turn, affects the performance of legacy relays as they depend on these characteristics to detect and protect against faults. One can expect increased levels of relay mis-operations as the penetration levels of renewables increase.

Another challenge is the fact that the transient response of the power system has become faster due to the presence of many power electronic systems. Legacy SCADA with data acquisition rates very slow for these transients is inadequate to address the new problems. It is important to use a much faster data acquisition system and commensurate protection and control technologies.

It is important to address these challenges. This paper presents a system that provides methods that are not affected by the changing characteristics of the power system and provides a fast response to the possible events in the power system.

\section{Objective of centralized substation protection}

Substation automation has been a continuous effort of the industry since the advent of computer technology. 
As technology advances, substation automation has been transforming into a more powerful but more complex task. Complexity generates risk. The risk can be mitigated with methods that have the capability to selfcheck and verify the various components of substation automation. The objective of this paper is to present technologies that enable full self-checking and verification of all the functions involved in substation operations, namely protection, control, and operation. This new approach is referred to as resilient Centralized Substation Protection, or rCSP. The objectives of rCSP are: (a) provide supervision of the protection and control function in a substation to ensure reliable performance of protection and control functions, (b) support the needs of the control center for substation visibility and awareness, (c) detect any anomalies in the substations operation including bad data, altered data, hidden failures, etc. and (d) detect in real-time any cyber-attacks. The first three items provide operational security, while the last ensures cyber security.

The system provides a validated model and current operating conditions, which constitutes the fundamental input for practically all the applications in a control center. In the paper, we discuss one application, optimal power flow, as an example.

The paper describes how each of these objectives is achieved with the proposed approach.

\section{Dynamic State Estimation protection}

\subsection{Description}

The Dynamic State Estimation Based Protection (EBP) method (a.k.a setting-less protection) is the basic building block of the centralized substation protection. The EBP was inspired by the differential protection method, which has been proved to be one of the most effective protection schemes without the need to be coordinated with any other protection functions [1]-[2]. Compared to the differential protection method, where the relay only monitors the validity of Kirchhoff's Current Law (KCL), EBP monitors the validity of all applicable physical laws in the protection zone, including KCL, Kirchhoff's Voltage Law (KVL), motion laws, thermodynamics laws and other depending on the type of the protection zone. EBP does not require complex settings and coordination with other protection functions. To implement EBP, three critical parts are required. (a) A high-fidelity dynamic model of the protection zone, (b) a set of measurements, and (c) a dynamic state estimation algorithm to monitor the operating conditions of the components within the protection zone. The three key parts of EBP will be briefly introduced in the following parts of this section, starting with the construction of the protection zone dynamic model.

As previously mentioned, a dynamic model of the protection zone is required for EBP. The dynamic model of a protection zone consists of models of each component within the protection zone. The first step of constructing the dynamic model of a specific component is to generate the mathematical model in terms of differential and algebraic equations that express the physical laws of the component. If there exist non-linear terms with order higher than two, then the model is quadratized by introducing additional state variables. The end result is a quadratized model which is cast into a standard format called Quadratized Device Model (QDM), shown below:

$$
\begin{aligned}
& i(t)=Y_{e q x 1} \mathbf{x}(t)+Y_{\text {equ } 1} \mathbf{u}(t)+D_{\text {eqxd } 1} \frac{d \mathbf{x}(t)}{d t}+C_{e q c 1} \\
& 0=Y_{\text {eqx } 2} \mathbf{x}(t)+Y_{\text {equ } 2} \mathbf{u}(t)+D_{\text {eqxd } 2} \frac{d \mathbf{x}(t)}{d t}+C_{e q c 2} \\
& \vdots \\
& 0=Y_{\text {eqx } 3} \mathbf{x}(t)+Y_{\text {equ } 3} \mathbf{u}(t)+\left\{\begin{array}{c}
\left.\mathbf{x}(t)^{T}\left\langle F_{\text {eqxx } 3}^{i}\right\rangle \mathbf{x}(t)\right\}+C_{\text {eqc3 }} \\
\vdots
\end{array}\right\}
\end{aligned}
$$

where $i(t)$ is the through variable vector of the model (terminal currents), $x(t)$ is the state variable vector of the model, Y, D, F are coefficient matrices, and $\mathrm{C}$ are constant terms in the model.

The QDM expressed with Equations (1) is integrated over the time intervals $[\mathrm{t}-2 \mathrm{~h}, \mathrm{t}]$ and $[\mathrm{t}-2 \mathrm{~h}, \mathrm{t}-\mathrm{h}]$ via quadratic integration, where $2 \mathrm{~h}$ is the time step. The result of the quadratic integration becomes the Algebraic Quadratic Companion Form (AQCF), shown as follows:

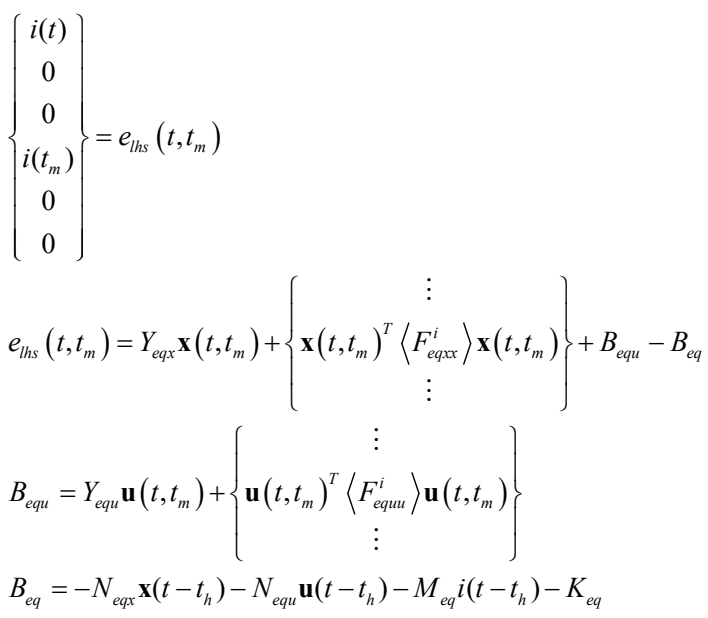

where $x\left(t, t_{m}\right)=\left[x(t), x\left(t_{m}\right)\right]$ and $i(t), i\left(t_{m}\right)$ are the state variable vector and through variable vector at times $t$ and $\mathrm{t}_{\mathrm{m}}$, respectively. $Y_{e q x}, F_{e q x}^{i}, N_{e q x}, M_{e q}$, and $K_{e q}$ are matrices calculated from the coefficient matrices in QDM. Details 
about the model quadratization process and quadratic integration were introduced in our previous publications, for example [3].

To obtain the AQCF model of the protection zone, the AQCF models of each component in the protection zone are first developed and subsequently are used to form the AQCF model for the entire protection zone. This process consists of the application of KCL at the interface nodes among components of the protection zone, which results in the elimination of through variables, and appending the internal equations of all components to the protection zone model.

The measurements are expressed as functions of the state of the protection zone. This is achieved by using the AQCF model of the protection zone to express each measurement by the model of the zone. Since the model is in the standard form shown in equation (2), the measurement model is also in the standard form shown as Equation (3), where $\mathrm{z}(\mathrm{t})$ are the measurements, $\mathrm{x}(\mathrm{t})$, $\mathrm{u}(\mathrm{t})$, and $\mathrm{i}(\mathrm{t})$ are the state variables, control variables and electric current variables of the protection zone, respectively.

$$
\begin{aligned}
& \mathbf{z}=Y_{m, x} \mathbf{x}+\left\{\begin{array}{c}
\vdots \\
\left.\mathbf{x}^{T} F_{m, x}^{i} \mathbf{x}\right\} \\
\vdots
\end{array}\right\}+Y_{m, u} \mathbf{u}+\left\{\begin{array}{c}
\vdots \\
\left.\mathbf{u}^{T} F_{m, u}^{i} \mathbf{u}\right\} \\
\vdots \\
C_{m}=N_{m, x} \mathbf{x}(t-h)+N_{m, u} \mathbf{u}(t-h)+M_{m} i(t-h)+K_{m}
\end{array}\right.
\end{aligned}
$$

The measurements are assumed to have been collected with PMUs, MUs, and/or PMU enabled numerical relays.

The dynamic state estimation uses the measurement model (3) and one of two options: (a) unconstrained optimization (weighted least squares approach) or (b) constraint optimization. The solution of the dynamic state estimation provides the estimates of the states, normalized residuals, the covariance of the state variable errors, and other metrics. An important part of the dynamic state estimation is the chi-square test which provides the probability that the measurements fit the model of the protection zone within the accuracy of the measurements. We refer to this probability as the "goodness of fit." This probability is also referred to as confidence level. A high confidence level (close to 1) indicates all the components in the protection zone are operating normally. A low confidence level (close to zero) indicates an abnormality within the protection zone, for example, a fault, a hidden failure, a cyberattack that altered data. The details about chi-square test calculations can be found in our previous publications [1], [2].

\subsection{Immunity to inverter effects}

As penetration levels of Distributed Energy Resources (DERs) in the power system increase, the characteristics of DERs are affecting the performance of power system protection. New challenges have emerged in this area, such as bidirectional power flow in distribution circuits, lower fault current levels, reduced negative and zero sequence currents during faults affecting the ability of relays to determine fault direction and others [4]. EBP is immune to these issues as it detects faults based on the overall consistency between the measurements and the dynamic model of the protection zone. So, changes in the characteristics and levels of fault currents will not affect the performance of EBP.

Distribution system protection schemes are dependent on the unidirectional power and current flow in classical distribution systems. The bidirectional power flows created by DERs cause issues to the conventional protection system. The combined current of forward and reverse power flow may be smaller than the protection setting threshold of the overcurrent protection. Large reverse currents due to DER may also trigger false tripping signals. With EBP, the tripping decision is made based on the consistency between the measurements and the protection zone model instead of overcurrent or direction of current flow at certain buses. The direction of the power flow will not affect the performance of the EBP relays.

Due to the complexity of the electric power system, the implementation of conventional protection schemes requires complex coordination between protection devices and various protection functions. With the proposed EBP scheme, there is no need to coordinate with other protection functions. EBP only needs a dynamic model of the protection zone and measurements. Also, the protection zone parameters can be estimated and corrected (fine-tuned) by the dynamic state estimation; specifically, the dynamic state estimation is modified to include as unknown states specific parameters that we need to fine-tune. In this case, the dynamic state estimation will provide the best estimate of the specific parameter(s).

Previous simulation results [5] showed that EBP outperformed other protection schemes in protecting distributed systems in many test cases. Overall, the proposed centralized substation protection scheme with EBP is reliable and effective for power systems with high penetration of inverter-interfaced resources.

\subsection{Model validation}

The accuracy and fidelity of the dynamic model are critical to the performance of the proposed EBP approach. For some components that are widely used in power system modeling, there already exist high-fidelity dynamic models. However, for some newly introduced components, especially inverters with proprietary manufacturer information, the modeling accuracy and 
fidelity may not be as high. Therefore, it is necessary to validate the protection zone model. One of the model validation approaches is hybrid dynamic simulation [6], where measured signals are applied to the protection zone model. The simulation results of the model are compared with actual measured data to validate the model. In the proposed EBP scheme, DSE is used to validate the protection zone model. As shown in Figure 1 , the field measurements and output from the dynamic model are used to calculate the consistency between the measurements and the model output. If the result shows a high confidence level, it means the measurements and model are validated. Vice versa, a low confidence level indicates that the measurements contain errors, or the model is not accurate, or both. Model validation with DSE requires accurate measurements from the field. Merging units are used to collect the measurements to avoid errors introduced by usually long cables in instrumentation channels and other instrumentation channel inaccuracies.

Parameter estimation also plays an important role in the model validation process. If the model is inaccurate initially, model parameters are fine-tuned based on the results of DSE. Parameter identification tasks are performed by setting some key model parameters as unknown states. [7] So, these key model parameters are fine-tuned with real-time field data to consistently improve the accuracy and fidelity of the dynamic model. The validated models of the power system components can also be used for other Energy Management System (EMS) applications.

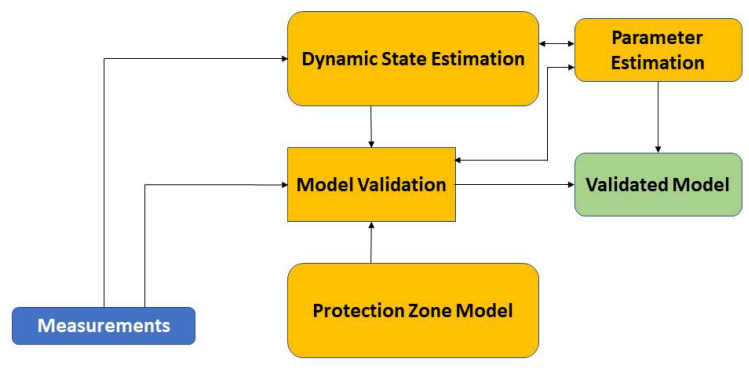

Figure 1: Illustration of protection zone model validation via DSE

\section{Protection system supervision}

In any engineering system that utilizes a data acquisition system to monitor and protect the system, the performance of the system becomes dependent on the validity of the measurements. Since the data acquisition systems can fail locally or in devices that process measurements or can be altered by a cyberattack on devices, it is important to develop methods that will be able to verify the validity and accuracy of the data. The proposed system uses dynamic state estimation to assess the validity and accuracy of the data by taking advantage of data redundancy at subsystem levels such as a substation, a distribution system, and other electrical installations. Data redundancy is defined as the ratio of the number of available data over the number of states of the system. In this section, we describe such an approach. The method is powerful in the sense that it does detect not only data anomalies but also identifies the root cause of the data anomalies. This is achieved by hypothesis testing, as described later.

\subsection{Substation Quasi-Dynamic State Estimator (sQSE)}

The database of setting-less relays, i.e., EBP relays, they can be utilized to autonomously create the substation QSE. Each EBP relay or other computing device converts the sampled values of voltage and currents into phasors; other sample values (speed, temperature, etc.) are decimated at the same rate as phasors. Subsequently, this data is streamed into the substation bus. Then, the phasor data and the models from the EBP relays are used to autonomously form the substation QSE. Hence, the following data are involved in the process of creating the substation QSE: (a) model of protection zone of the EBP (once and by exception if there is a model change, for example, a tap change for a transformer), (b) raw and estimated values of measurements, (c) model of measurements in objectoriented format, and (d) status of breakers and switches within the protection zone of the EBP. Figure 1 summarizes the overall process of forming the substation QSE.

The substation QSE enables a centralized protection scheme that ensures the validity and trustworthiness of the data. In case of abnormalities, the centralized substation protection identifies the cause of the abnormality (hidden failures, cyber-attacks, etc.), corrects and replaces the corrupted data so that the setting-less relays will operate with corrected data, and will take the correct protection decisions. In other words, the analytics determine with computable certainty the following: (a) all data are valid, i.e., no instrumentation errors, no hidden failures, no data attacks, or (b) bad data are present, in which case an identification process is initiated to determine the root cause of the bad data. The identification process is described next.

\subsection{Anomaly detection and identification}

One of the main functionalities provided by substation QSE is the ability for anomaly detection and identification. The QSE computes the best estimate of 
the substation's dynamic state. Subsequently, the goodness of fit between the measurements and the substation model is computed via the well-known chisquare test, which calculates the probability of goodness of fit between the measurement and the substation model. This probability is also referred to as confidence level. A high probability of goodness of fit, i.e., more than 0.90 , results in declaring all the data in the substation valid. In this case, no further action is required. Otherwise, there is an anomaly in the data, and the root cause of the anomaly must be identified. For this purpose, a series of hypothesis testing is performed to identify the root cause of the bad data, i.e., hidden failures, bad IED settings, power fault, data alteration due to a cyber-attack, etc. Once the root cause is identified, the compromised data is known; this data can be replaced with estimated values using the validated model of the system and the operating condition.

The aim of hypothesis testing is to identify the cause of data abnormalities in real-time and replace the compromised data with estimated values. The mechanics of hypothesis testing are as follows: First, suspect measurements are identified from normalized residuals, which are provided by the QSE. Second, suspect measurements are grouped using the source of the data (merging units) and also by the protection zone. Third, protection zones are identified for which the protection functions have reported a fault. These classifications lead to specific hypothesis tests. We are discussing below examples of hypothesis testing.

Hypothesis Type 1 (H1): Remove suspect measurements and rerun QSE. If the probability is high (more than 0.9), then: (a) removed measurements are bad, (b) the root cause is at the devices reporting these measurements, (c) issue diagnostics, and (d) replace bad data with estimated values. End hypothesis testing. Otherwise, go to $\mathrm{H} 2$.

Hypothesis Type 2 (H2): (determine if a fault decision is correct). For the reported faulted device, remove all internal device measurements and remove the faulted device model from the substation model. Then rerun QSE. If the probability is high (more than 0.9), then: (a) the device/protection zone is truly experiencing an internal fault. Allow zone relay to trip the faulted device. End hypothesis testing.

Hypothesis Type 3 (H3): This test combines type 1 and type 2 hypothesis testing to cover the case of a simultaneous fault and a hidden failure.

Note that hypothesis testing continues until the root cause has been identified. Then the compromised data are replaced with estimated values which are computed with the validated model and the estimated state of the system.

It is important to note that the reliability of the proposed hypothesis testing is possible because of the large redundancy of measurements at the substation level. Redundancy is defined as the number of measurements available over the number of states needed to describe the system.

\subsection{Data correction}

In case the identified root cause is compromised data (from hidden failures, equipment malfunctioning, data alteration), the data correction/replacement process is executed. The hypothesis testing has identified a number of compromised measurements. Then using the model of the entire substation and the real-time operating conditions (both provided by the substation QSE), the physical quantities represented by the compromised measurements are computed (estimated values). The computed quantities are streamed into the process bus, replacing the actual data collected by the merging units. Hence, any computing device using the sampled values at the process bus will now be using corrected data. At the same time, the system issues a message to the operator for repairing the system and alleviating the source of the compromised data.

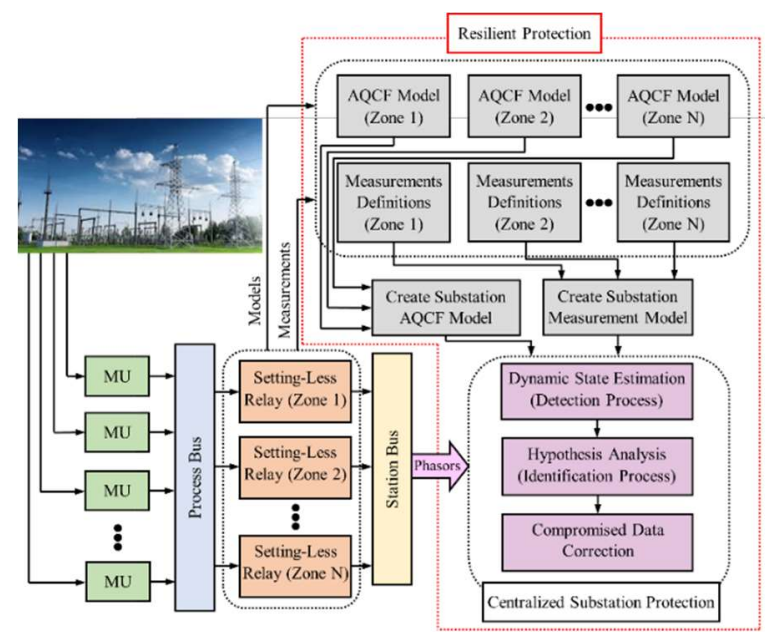

Figure 2. Overview of resilient protection approach

\subsection{Resilient protection under failures}

According to the US Presidential Policy Directives21 (PPD-21), resilience is the ability to prepare for and adapt to changing conditions and withstand and recover rapidly from disruptions. The disruptions include both physical disruptions and cyber disruptions [13]. Implementation of the proposed schemes creates a new paradigm for resiliency in protection systems with the capability of correcting compromised data (selfhealing), as shown in Figure 2. The proposed resilient protection scheme supervises all the setting-less relays in a substation in order to maintain the secure operation 
of the system under failures. As illustrated in Figure 2, measurements are streaming from merging units into the setting-less relays through the process bus. In addition to being used by setting-less relays for the protection of each zone, these data are utilized to obtain the phasor quantities. These phasors are used to form the substation QSE, and consequently, ensure the data validity via the anomaly detection and identification process. If an abnormality is detected, hypothesis testing is performed to identify if the root cause of the abnormality. If compromised data have been identified, the compromised data are replaced with its best estimate obtained from the substation-wide state estimator. The relays will continue to perform their protective functions with validated data. In this way, the protection and control system is self-healed against the abnormality. Since compromised input data into a relay typically results in mis-operations, the proposed system ensures that the protection and control system continues to operate reliably until the root cause is repaired.

In case of cyber disruptions, alteration or loss of measurements scenarios is handled the same way as any other source of anomaly. In terms of communication failures, it is important to note that most systems have redundant communications for reliability. Thus, the probability of communication loss is very low. While the protection and control system can revert to methods that are based only on local data, this topic is beyond the scope of this paper - also due to lack of space to cover this issue [14], [15].

The proposed approach for a resilient protection and control system for substations introduces numerous advantages over the conventional methods. The dynamic state estimation at the protection zone level eliminates the need for coordinating multiple complex protection schemes. This results in a much simpler protection scheme from the user's point of view. Furthermore, it is much faster as it detects faulty conditions within a few measurement samples (submillisecond response). Tripping, if warranted, is controlled by user-selected parameters (for example, a protection engineer may want to trip with a delay of 3 cycles). It is more sensitive than the present technology as it can detect faults that legacy systems miss, such as high impedance faults and faults near neutrals. The proposed protection scheme is well suited for the new hardware approaches for substation protection and control, namely the use of merging units. Maintenance, calibration, and testing can be fully automated with appropriate software, leading to a further decrease in the substation operating cost [16].

\section{Application}

Several important power systems operation and control applications are needed to maintain the safe and reliable operation of the grid. Some of these applications are load forecasting, optimization (ED and OPF), available transfer capability, security assessment, congestion management, visualizations, etc. The availability of a validated real-time model of the system enables these applications. The proposed scheme, i.e., Centralized Substation Protection (CSP), provides a validated real-time model and allows for the seamless integration of traditional and new applications. In previous publications, we discussed integrations of traditional applications such as stability monitoring and volt/var control [7], as well as new applications such as the Reserve-O-Meter (RoM), which is an application that evaluates the available reserves from each customer and utility owned resources along the distribution feeder [8]. The focus of this paper is on the following three applications: Control Center Operation, Optimal Power Flow and Operations Planning.

\subsection{Control center operations}

The control center is the centralized higher-level location for monitoring, control, and operation of the electricity grid. Functions of a control center can be grouped into (a) Supervisory Control and Data Acquisition (SCADA), (b) energy management and optimization system, and (c) security and operational reliability functions.

Data Acquisition (DA) comprises the collection of data at remote equipment in the field and gathered at the control center. The data consist of status variables, current and/or power flow measurements, voltage magnitude, and phase measurements. These measurements are used for further application. Supervisory Control (SC) is the system responsible for the visualization of the state of the system as well as the ability to control remote devices. The proposed system provides the next generation of SCADA since it provides data at rates of once per cycle (two orders of magnitude faster than legacy SCADA).

The Energy Management and Optimization System functions depend on the real-time model of the system. These functions can be grouped into (a) wide-area realtime model building and (b) optimization and energy management applications, such as economic dispatch, optimal power flow, scheduling functions, and others.

The security and operational reliability functions are the "watchdog" of the system, which include security assessment, transient stability monitoring, voltage stability monitoring, and others. 
The real-time model is constructed from the data provided by the proposed system, i.e., the new SCADA streaming into the control center at a rate of once per cycle. Status variables are used to determine the configuration of the network and other data is used to estimate the actual operating conditions of the system. Security assessment can be performed at user-selected intervals; when it is initiated, it will use the current validated model of the system.

CSP not only improves substation protection but also provides the following benefits for the operations of the control centers:

- Operations at higher temporal resolution: The realtime data granularity of today's control center operation is defined as $2 \mathrm{~s}$ or so [11]. CSP allows for real-time to be defined as 1 cycle.

- Real-time validated model availability: CSP provides a real-time validated model for each substation updated. The wide area network model is synthesized as discussed in the following subsection.

- Prevention of cascading outages: The current industry practice, i.e., not having a validated model as well as operation a higher time granularity at the control center and a lower time granularity for the protection system could result in cascading outages such as the North American and Italian blackouts of 2003 [11]. CSP closes this gap and allows for the prevention of cascading outages.

- Better and unprecedented supervisory control abilities: Today's supervisory control (SC) is mostly manual, i.e., the operator issues commands; however, CSP allows for total or partial automation of SC. CSP provides corrected data to the control center, allowing for the display of correct and uncompromised data. Which in turn, allows the operator to correctly visualize the system and take appropriate decisions in partially automated SC. As part of anomaly detection, CSP issues diagnostics/alarms that are displayed at the control center when it identifies that correction of the root cause of bad data requires human involvement (for example, a blown fuse).

- Reliable DSA: CSP provides a validated real-time dynamic model of the system. A dynamic model of the system can be used to reliably perform DSA.

- Robust modern ACG: Inverter-based resources (IBRs) pose a challenge for net interchange control, but an AGC based on the proposed method can address this challenge but providing control at much faster rates.

In subsequent paragraphs, we discuss how the proposed rCSP system provides robust solutions and changes the paradigm of power system operations.

\subsection{System-Wide QSE (wQSE)}

The results provided by the substation-wide state estimation can be utilized to facilitate a system-wide QSE. To this end, the validated and time-tagged results from all substation QSEs are streamed into the control center. At the control center, the synthesize the operating conditions of the entire system are synthesized by putting together the state estimates of all substations of the same time tag, see Figure 3. In order to facilitate efficient communications, each substation sends only its real-time model to the control center, which comprises a very small amount of data. In case the connectivity changes, the connectivity data are transmitted by exception. Similarly, in case of a change in model, the new mathematical model will be transmitted by exception.

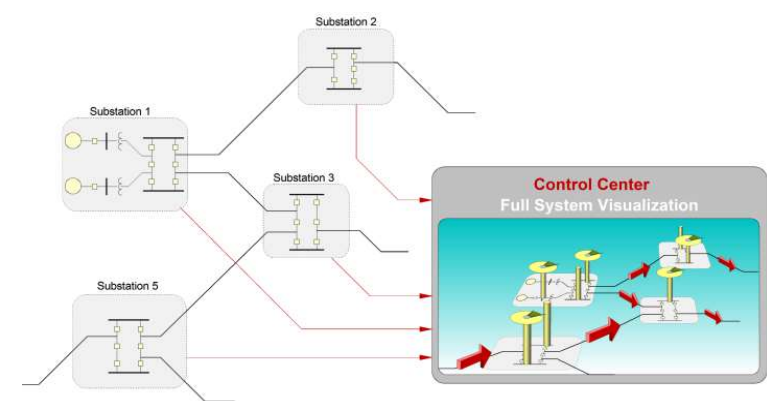

Figure 3. Synthesis of system-wide QSE from substation QSEs

Wide-Area Network Synthetization: CSP allows for seamless building of the validated wide-area network model from substation models, as illustrated in Figure 4. The substation model objects are, in turn, built from device model objects. Device models objects are constructed as follows: (1) the set of equalities and inequalities describing the law that governs the operation of the device are derived, (2) these algebraic and differential equations are quadratized so that the highest level of nonlinearity is 2, resulting in a model referred to as the Quadratized Device Model (QDM), (3) the QDM is integrated using quadratic integration to obtain a purely algebraic model, referred to as the State \& Control Algebraic Quadratic Companion Form (SCAQCF).

The SCAQCF object, which is presented in previous publications [1-4], is of the form: 


$$
\begin{aligned}
& \left\{\begin{array}{l}
g_{1} \\
g_{2} \\
g_{3} \\
g_{4} \\
g_{5} \\
g_{6}
\end{array}\right\}:\left\{\begin{array}{c}
\mathbf{i}(t) \\
0 \\
0 \\
\mathbf{i}\left(t_{m}\right) \\
0 \\
0
\end{array}\right\}=Y_{x} \mathbf{x}+Y_{u} \mathbf{u}+\left\{\begin{array}{c}
\vdots \\
\mathbf{x}^{T} F_{x x}^{k} \mathbf{x} \\
\vdots
\end{array}\right\}+\left\{\begin{array}{c}
\vdots \\
\left.\mathbf{u}^{T} F_{u u}^{k} \mathbf{u}\right\} \\
\vdots
\end{array}\right\}+\left\{\begin{array}{c}
\vdots \\
\left.\mathbf{u}^{T} F_{e q u x}^{k} \mathbf{x}\right\} \\
\vdots
\end{array}\right\}-B \\
& B=-N_{x} \mathbf{x}(t-h)-N_{u} \mathbf{u}(t-h)-M(t-h)-K
\end{aligned}
$$$$
\mathbf{h}(\mathbf{x}, \mathbf{u})=Y_{f x} \mathbf{x}+Y_{f u} \mathbf{u}+\left\{\begin{array}{c}
\vdots \\
\mathbf{x}^{T} F_{f x x}^{i} \mathbf{x} \\
\vdots
\end{array}\right\}+\left\{\begin{array}{c}
\vdots \\
\mathbf{u}^{T} F_{f u u u}^{i} \mathbf{u} \\
\vdots
\end{array}\right\}+\left\{\begin{array}{c}
\vdots \\
\mathbf{u}^{T} F_{f u x}^{i} \mathbf{x} \\
\vdots
\end{array}\right\}+C_{f c} \leq 0
$$$$
\mathbf{u}_{\text {hmin }} \leq \mathbf{u}(t) \leq \mathbf{u}_{h \max }
$$

with $\mathbf{x}=\left[\begin{array}{ll}\mathbf{x}(t) & \mathbf{x}\left(t_{m}\right)\end{array}\right]^{T}$ and $\mathbf{u}=\left[\begin{array}{ll}\mathbf{u}(t) & \mathbf{u}\left(t_{m}\right)\end{array}\right]^{T}$

Where $g_{l}$ and $g_{4}$ are terminal equations and $g_{2}, g_{3}, g_{5}$, and $g_{6}$ are internal equations. $t$ and $t_{m}$ respectively denote the present time and midpoint between the present time and the previous time $t-t_{h}$. Vectors $\mathbf{i}, \mathbf{x}, \mathbf{u}$, are respectively the vectors of through, state, control variables. Matrices F and $\mathrm{Y}$ contain respectively linear and quadratic coefficients of variables at present time $t$. Matrix B contains past history terms, and matrices $\mathrm{N}$ and $\mathrm{K}$ are linear coefficients of variables at the previous time $t-t_{h}$.

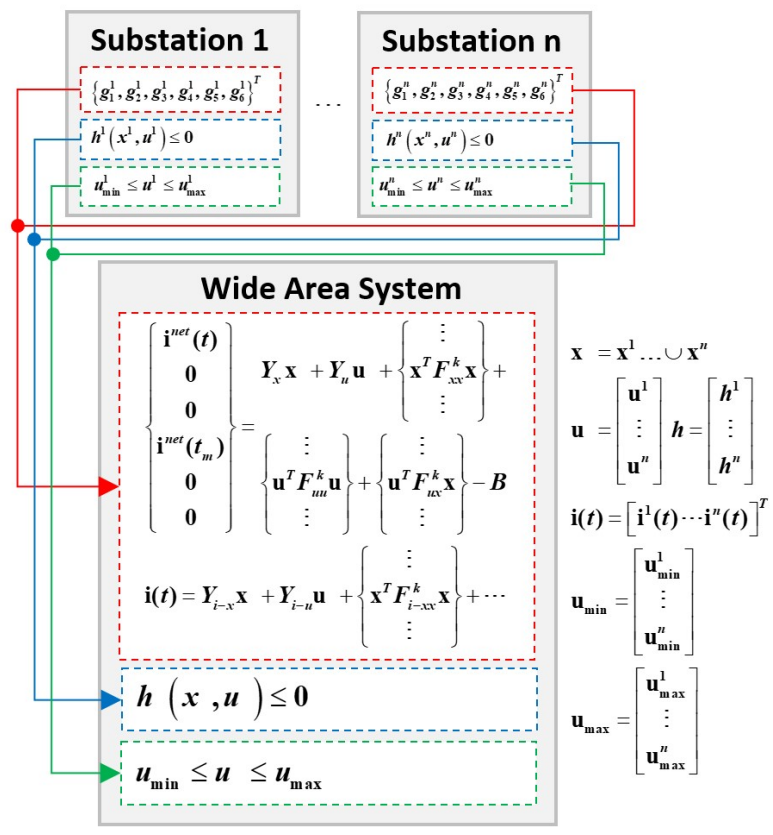

Figure 4. Wide Area System model building

Given a wide area that is made up of $n$ substation equipped with CSP, using CSP results, the network model is synthesized as follows: interface equations at the terminal of the wide-area network are defined as illustrated in Figure 4; generalized Kirchhoff's Current Law is applied at all nodes in the network, substation internal equations, functional constraints and controls are stacked to form the wide-area internal equations, functional constraints, and controls, and state vector is the union of the state vectors of substations [7][12]. The end result is the system-wide network model that can be used for practically any application in the control center.

Two scheduling functions, namely Optimal Power Flow and Operational Planning, are discussed next as examples of utilization of the network model provided by the rCSP.

\subsection{Optimal Power Flow}

The validated models from substations rCSPs can be utilized to optimize the grid's operating conditions, determining the control actions needed to achieve a selected objective such as minimization of area-wide generation cost, levelization of voltage profile, levelization of flow in key circuits, etc. The Optimal Power Flow (OPF) is the tool of choice to solve these optimization problems. Any OPF problem can be expressed in its simplest form as:

$$
\begin{array}{ll}
\min & J(\mathbf{x}, \mathbf{u}) \\
\text { s.t. } & g(\mathbf{x}, \mathbf{u})=0 \\
& h(\mathbf{x}, \mathbf{u}) \leq 0 \\
& \mathbf{u}_{\min } \leq \mathbf{u} \leq \mathbf{u}_{\max }
\end{array}
$$

Where $\quad J(\mathbf{x}, \mathbf{u}), g(\mathbf{x}, \mathbf{u})$, and $h(\mathbf{x}, \mathbf{u})$ denote the objective function, the power flow equations, and the functional constraints. All are functions of states $\mathbf{X}$ and controls $\mathbf{u}$.

The Quadratic OPF (QOPF) formulation in SCAQCF is invariant whether the system is the positive sequence or three-phase modeling due to the use of the object-oriented modeling approach. This formulation is expressed as:

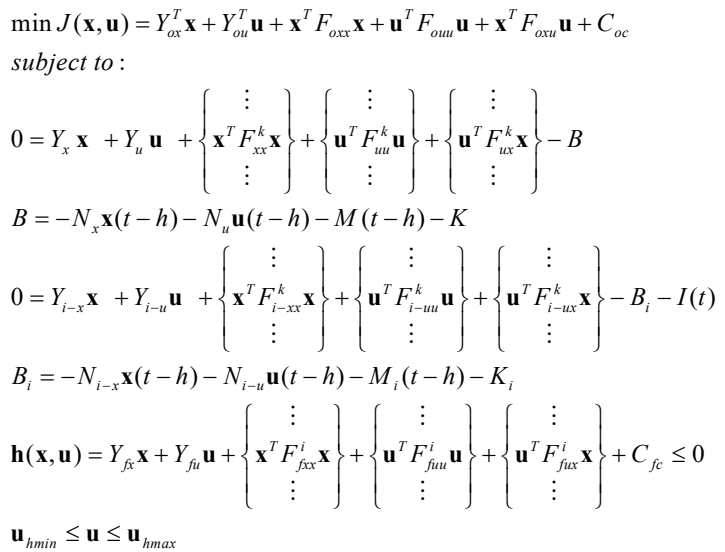

Network through a variable $\mathbf{i}^{\text {net }}$ is a zero vector since the network for OPF purposes is a closed network. 
The problem can then be solved using the Convex Solution - Sequential Linear Programming (CS-SLP) algorithm. The flow chart for CS-SLP is depicted in Figure 5. This algorithm is an extension of the Sequential Linear Programming (SLP) algorithm that has been presented in previous publications. In this algorithm, the QOPF is initially convexified, and the resulting convex problem is solved to provide a warm start for SLP. $n_{c s}$ is the total number of convexification iterations allowed. Note that if this number is set to 0 , the algorithm simply becomes an SLP algorithm.

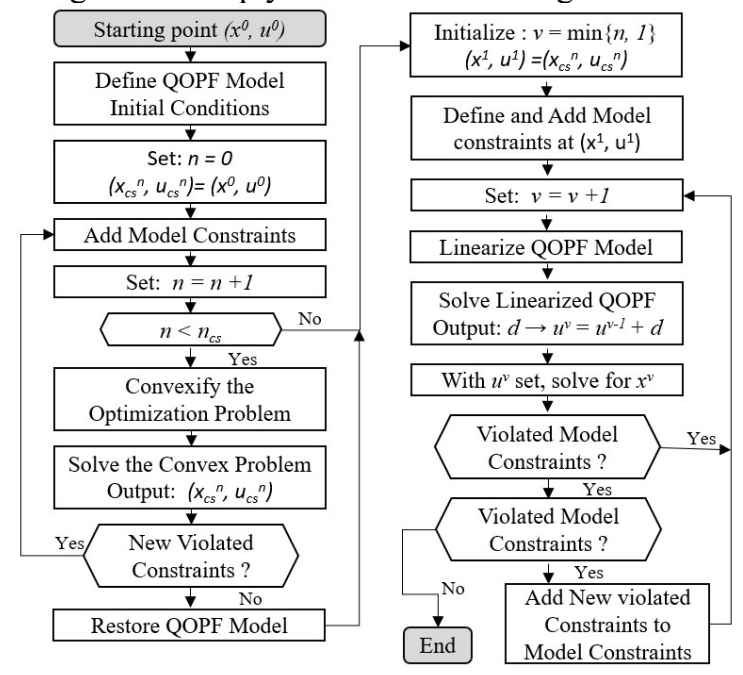

\section{Figure 5. Flowchart of CS-SLP algorithm for OPF solution}

The cost minimization OPF was solved with CSSLP for 3 test systems, and the results are reported in Table 1 . The three systems are a small four bus system and two network models from the ARPA-E Go competition: Network 01R-10 and Network_02*-173. In all three cases, the number of convex iterations is set to 1 , i.e., $n_{c s}=1$.

Table 1. Major metrics of QOPF solution with CSSLP

\begin{tabular}{l|c|c|c|c}
\hline $\begin{array}{l}\text { Number } \\
\text { of SLP } \\
\text { Iterations }\end{array}$ & $\begin{array}{c}\text { Number of } \\
\text { Model } \\
\text { Constraints }\end{array}$ & $\begin{array}{c}\text { Number of } \\
\text { Active } \\
\text { Constraints }\end{array}$ & $\begin{array}{c}\text { Convex } \\
\text { Problem } \\
\text { Obj. Val. } \\
(\$)\end{array}$ & $\begin{array}{c}\text { Objective } \\
\text { Function } \\
\text { Value (\$) }\end{array}$ \\
\hline \hline
\end{tabular}

Small System 1

( 4 buses, 2 generators, 1 transformer, 3 lines, 1 load)

\begin{tabular}{c|c|c|c|c}
\hline 6 & 5 & 3 & 6308.51 & 6612.85 \\
\hline \hline \multicolumn{5}{c}{ Small System 2 }
\end{tabular}

(14 buses, 5 generators, 3 transformers, 17 lines, 11 loads, 1 fixed

\begin{tabular}{c|c|c|c|c}
\multicolumn{5}{c}{ shunt) } \\
\hline 3 & 4 & 3 & $13,964.21$ & $14,686.15$ \\
\hline \hline
\end{tabular}

(500 buses, 224 generators, 193 transformers, 540 lines, 281 loads, 5 fixed shunts, 31 switched shunt)

\begin{tabular}{|l|c|c|c|c|}
6 & 113 & 53 & $271,401.97$ & $273,289.62$ \\
\hline
\end{tabular}

\subsection{Operations Planning}

Operations Planning (OP) is concerned with solving an optimization problem over a horizon period. OP problems can be unit commitment, flexibility/storage evaluation, and planning, etc. This paper focuses on the Multi-Stage Quadratic Flexible Optimal Power Flow (MQFOPF) proposed within the platform of the rCSP.

The MQFOPF is equivalent to summing the objective function at all stages and stacking up the constraints form each stage in the planning horizon. It is of the form:

$$
\begin{array}{ll}
\min & \sum_{i=1}^{H} J\left(\mathbf{x}_{i}, \mathbf{u}_{i}\right) \\
\text { s.t. } & g_{i}\left(\mathbf{x}_{i-1}, \mathbf{x}_{i}, \mathbf{u}_{i}\right)=0 \quad i=1,2, \ldots, H \\
& h_{i}\left(\mathbf{x}_{i-1}, \mathbf{x}_{i}, \mathbf{u}\right) \leq 0 \quad i=1,2, \ldots, H \\
& \mathbf{u}_{i, \min } \leq \mathbf{u} \leq \mathbf{u}_{i, \max } \quad i=1,2, \ldots, H
\end{array}
$$

Where subscript $i$ denotes functions and vectors at stage $i$, subscript $i-1$ denotes the previous stage, and $H$ is the number of stages in the horizon period.

The generalized SCAQCF formulation for the $\mathrm{MQFOPF}$ is given as:

$$
\begin{aligned}
& \min J(\mathbf{x}, \mathbf{u})=Y_{\text {Mox }}^{T} \mathbf{x}+Y_{\text {Mou }}^{T} \mathbf{u}+\mathbf{x}^{T} F_{\text {Moxx }} \mathbf{x}+\mathbf{u}^{T} F_{\text {Mout }} \mathbf{u}+\mathbf{x}^{T} F_{\text {Moxu }} \mathbf{u}+C_{\text {Moc }} \\
& \text { subject to : } \\
& g(\mathbf{x}, \mathbf{u})=Y_{M x} \mathbf{x}+Y_{M u} \mathbf{u}+\left\{\begin{array}{c}
\vdots \\
\mathbf{x}^{T} F_{M x x}^{k} \mathbf{x} \\
\vdots
\end{array}\right\}+\left\{\begin{array}{c}
\vdots \\
\mathbf{u}^{T} F_{M u u}^{k} \mathbf{u} \\
\vdots
\end{array}\right\}+\left\{\begin{array}{c}
\vdots \\
\mathbf{u}^{T} F_{M u x}^{k} \mathbf{x} \\
\vdots
\end{array}\right\}-C_{M}
\end{aligned}
$$

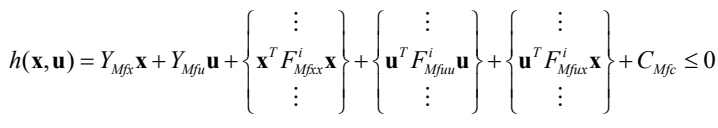

$$
\begin{aligned}
& \mathbf{u}_{\text {hmin }} \leq \mathbf{u} \leq \mathbf{u}_{\text {hmax }}
\end{aligned}
$$

Where subscript $M$ denotes that this is a multi-stage problem, $\mathbf{x}=\left[\begin{array}{lllllll}\mathbf{x}\left(t_{1}\right) & \mathbf{x}\left(t_{1, m}\right) & I\left(t_{1}\right) & \cdots & \mathbf{x}\left(t_{H}\right) & \mathbf{x}\left(t_{H, m}\right) & I\left(t_{H}\right)\end{array}\right]^{T}$, $\mathbf{u}=\left[\begin{array}{lllll}\mathbf{u}\left(t_{1}\right) & \mathbf{u}\left(t_{1, m}\right) & \cdots & \mathbf{u}\left(t_{H}\right) & \mathbf{u}\left(t_{H, m}\right)\end{array}\right]^{T}$

Similar to the single-stage OPF, this problem is solved using the CS-SLP algorithm, for which the flowchart is depicted in Figure 2.

\section{Conclusions}

This paper presented a new paradigm for the protection, control, operation, and optimization of an electric energy system. The new model starts at the substation and uses dynamic state estimation protection, which has the following advantages relative to the new challenges: protection functions immunity to the changing characteristics of power systems such as reduced fault currents, reduced negative and zero sequence fault currents, bilateral follow of fault current and power flow in distribution systems, sub-millisecond detection times and other attractive characteristics, and 
ability to validate the models of protection zones. In addition, the estimation-based protection provides data and models to any other level of the system to support the needs and applications in these levels. At the same time, we proposed a substation-based estimation method to supervise the data validity, detect data anomalies, identify the source of data anomalies and take corrective action. The integration of the estimation-based protection functions and the substation state estimator provides a system that continuously validates data and models and, in case of anomalies, has the capability to self-heal by correcting bad data. The overall system streams validated data and models to the control center to support system-wide applications. In the paper, we discussed several applications and focused on the important application of optimal power flow. The proposed models enable innovative methods for the formulation and solution of the optimal power flow. Specifically, a generalized method to convexify the optimal power flow into a convex quadratic model for which robust and fast solvers exist. The final solution of the OPF problem is obtained with SLP starting from the convex model and using the unrelaxed OPF model. The proposed approaches favorably address the challenges posed by the present trajectory of the power system.

\section{References}

[1] A. P. S. Meliopoulos, G. J. Cokkinides, Z. Tan, S. Choi, Y. Lee, and P. Myrda, "Setting-Less Protection: Feasibility Study," 2013 46th Hawaii International Conference on System Sciences, 2013.

[2] A. P. Meliopoulos, G. J. Cokkinides, P. Myrda, Y. Liu, R. Fan, L. Sun, R. Huang, and Z. Tan, "Dynamic State Estimation-Based Protection: Status and Promise," IEEE Transactions on Power Delivery, vol. 32, no. 1, pp. 320 330, 2017.

[3] A. P. S. Meliopoulos, G. J. Cokkinides, and G. K. Stefopoulos. "Quadratic integration method." Proceedings of the 2005 International Power System Transients Conference (IPST 2005). 2005.

[4] V. Telukunta, J. Pradhan, A. Agrawal, M. Singh, and S. G. Srivani, "Protection challenges under bulk penetration of renewable energy resources in power systems: A review," CSEE Journal of Power and Energy Systems, vol. 3, no. 4, pp. 365-379, 2017.

[5] K. Liu, A. P. Sakis Meliopoulos, B. Xie, C. Zhong, and J. Xie, "Dynamic State Estimation-Based Protection of Distribution Systems with High Penetration of DERs,"
2020 IEEE Power \& Energy Society General Meeting (PESGM), 2020.

[6] Z. Huang, T. Nguyen, D. Kosterev, and R. Guttromson, "Model Validation of Power System Components Using Hybrid Dynamic Simulation," 2005/2006 PES TD.

[7] A. P. Meliopoulos, G. J. Cokkinides, S. Grijalva, R. Huang, E. Polymeneas, Paul Myrda, and Mel Gehrs, "Integration \& Automation: From Protection to Advanced Energy Management Systems", Proceedings of the 2013 IREP Symposium on Bulk Power System Dynamics and Control, pp 1-13, Rethymnon, Greece, Aug. 25-30, 2013.

[8] A. P. S. Meliopoulos, E. Polymeneas, Z. Tan, R. Huang and D. Zhao, "Advanced Distribution Management System," in IEEE Transactions on Smart Grid, vol. 4, no. 4 pp. 2109-2117, Dec. 2013, doi: 10.1109/TSG.2013.2261564.

[9] E. Polymeneas and A. P. Sakis Meliopoulos, "MarginBased Framework for Online Contingency Selection in Unbalanced Networks," in IEEE Transactions on Power Systems, vol. 32, no. 1, pp. 30-38, Jan. 2017, doi: 10.1109/TPWRS.2016.2555987.

[10] J. Zhao et al., "Power System Dynamic State Estimation: Motivations, Definitions, Methodologies, and Future Work," in IEEE Transactions on Power Systems, vol. 34, no. 4, pp. 3188-3198, July 2019, doi: 10.1109/TPWRS.2019.2894769.

[11] F. F. Wu, K. Moslehi and A. Bose, "Power System Control Centers: Past, Present, and Future," in Proceedings of the IEEE, vol. 93, no. 11, pp. 18901908, Nov. 2005, doi: 10.1109/JPROC.2005.857499

[12] A. P. Meliopoulos, G. J. Cokkinides, R. Huang, E. Polymeneas, and Paul Myrda, "Grid Modernization: Seamless Integration of Protection, Optimization \& Control", Proceedings of the of the 47th Annual Hawaii International Conference on System Sciences, Hawaii, HI, Jan. 6-9, 2014.

[13] Linkov, Igor, Daniel A. Eisenberg, Kenton Plourde, Thomas P. Seager, Julia Allen, and Alex Kott. "Resilience Metrics for Cyber Systems." Environment Systems and Decisions 33, no. 4 (2013): 471-76. https://doi.org/10.1007/s10669-013-9485-y.

[14] Leszczyna, Rafał. "A Review of Standards with Cybersecurity Requirements for Smart Grid." Computers $\begin{array}{llll}\& & \text { Security } 77 & \text { (2018): } & \text { 262-76. }\end{array}$ https://doi.org/10.1016/j.cose.2018.03.011.

[15] Lin, Hua, Santosh Sambamoorthy, Sandeep Shukla, James Thorp, and Lamine Mili. "A study of communication and power system infrastructure interdependence on PMU-based wide area monitoring and protection." In 2012 IEEE Power and Energy Society General Meeting, pp. 1-7. IEEE, 2012.

[16] CIGRE WG B5.53 Report, "Test strategy for Protection, Automation and Control (PAC) functions in a fully digital substation based on IEC 61850 applications", Reference: 760, March 2019. 